\title{
Sunk Cost Efficiency with Discrete Competitors ${ }^{1}$
}

\author{
Linus Wilson \\ Assistant Professor of Finance \\ University of Louisiana at Lafayette \\ B. I. Moody III College of Business \\ Department of Economics \& Finance \\ 214 Hebrard Boulevard, Moody Hall 326 \\ P. O. Box 44570 \\ Lafayette, LA 70504-4570 \\ Phone: (337) 482-6209 \\ Fax: (337) 482-6675 \\ Email: linuswilson [at] louisiana [dot] edu \\ Web: http://www.linuswilson.com
}

First Draft: May 2006

This Draft: January 2009

${ }^{1}$ An earlier draft of this paper was circulated as “Sunk Cost Efficiency with Identical Competitors.” 


\title{
Sunk Cost Efficiency with Discrete Competitors
}

\author{
Abstract \\ When entrants only differ in their exogenous entry costs, the order in which potential \\ firms enter does not affect industry size. With discrete competitors, entry orderings \\ can affect total sunk costs and the identity of entrants. A necessary and sufficient \\ condition is established for sunk, entry costs in the industry to be minimized, \\ regardless of entry ordering.
}

Journal of Economic Literature Classifications: $\quad$ L11 \& L13

Keywords: $\quad$ Sunk Costs, Entry, Market Structure 


\section{Introduction}

Different entrepreneurs may have more or less familiarity with setting up a storefront or complying with local regulations that pertain to their business. These entry cost advantages do not necessarily lead to variable cost advantages. Managers

of rival firms face the same labor, raw materials, and energy costs of their competitors. Therefore, it seems that heterogeneity in sunk, entry costs and nearly identical variable costs are common in many business sectors.

New businesses form in dribs and drabs for the most part. One entrepreneur or firm decides to bring a business into being on a different day, month, or year than the next competitor. Therefore, a model of entry into an industry is best modeled as a sequential game. This paper attempts to find out under what circumstances path dependence — the ordering of entry — can affect the efficiency of the industry (the industry’s aggregate sunk costs.) This paper is more general than the standard approach of assuming that sunk and variable costs are identical. Instead, this paper assumes that there is some exogenous heterogeneity in the sunk costs of potential entrants. In this paper, potential competitors play a free entry game where the sequence of potential entrants is common knowledge. Each firm is given the opportunity to pay their entry cost or stay out of the industry forever. Then the firms compete and collect their profits before investment costs in the last stage.

With identical variable costs, the exogenous heterogeneity in sunk costs of entry does not improve a firm’s ability to compete in the final stage of the game. 
Instead, lower setup costs make entry more attractive (and socially efficient) for some firms.

We will see that the sequencing of entry decisions does not affect the size of the industry. Yet, the ordering of entry potentially affects the magnitude of entry costs incurred. This study finds the necessary and sufficient condition for fixed entry costs to be minimized for a given industry size, regardless of entry ordering. When the necessary and sufficient condition is satisfied, only the lowest sunk cost firms will enter. This is the first and only study to derive sufficient conditions for sunk costs to be minimized regardless of entry orderings when there are discrete competitors with identical variable cost functions.

The author knows of no other model of this type. The model is primarily distinguished by its exogenous heterogeneity in firms' entry costs. Studies either assume that entry costs are identical, as in Mankiw and Whinston (1986), or that sunk costs are endogenous. Examples of the latter approach are Spence (1977) and Dixit (1980) where the first mover chooses its variable costs to deter or accommodate further entry.

A more recent paper by Roberts (2007) endogenizes the opportunity cost of entry. This distinguishes it from the present approach. Further, the model of Roberts (2007) assumes that entry decisions are taken simultaneously. In contrast, this paper assumes that entry decisions are sequential. Many of the inefficiencies in Roberts (2007) disappear when sequential entry decisions are made in this paper. The relative efficiency of the sequential results here when compared to the results in Roberts' (2007) simultaneous move game is not surprising. Mixed strategy equilibria found in 
simultaneous move games often lead to inefficiencies even when players wish to behave cooperatively.

Ghemwhat and Nalebuff (1985) have a model of exit in a declining industry where firms must pay rent on their capital to remain in the industry. Despite this obvious difference, there are some analogies between models of entry an exit. The rent on capital in Ghemwhat and Nalebuff (1985) can be viewed as a sunk cost of entering the industry. Ghemwhat and Nalebuff (1985) find that firms which pay higher costs of staying in the industry are the first firms exit first in a declining industry. This is analogous to this paper's result that, under some circumstances, regardless of entry ordering only the lowest sunk cost firms will enter the industry. Yet, there is an important difference between this study and Ghemwhat and Nalebuff (1985). That study assumes that higher sunk costs of staying in the industry are associated with greater capacity and market share. Here we assume that higher sunk entry costs are exogenous and unrelated to variable costs and thus the firm's market share. Ghemwhat and Nalebuff (1985) as do many other studies of entry and exit assume for a given unit of capacity (or market share) that sunk costs identical. Unlike Ghemwhat and Nalebuff (1985), in the present paper, the sunk cost of entry per unit of market share is different for every potential entrant.

Wilson (2009) is similar to this study. As does this paper, Wilson (2009) assumes that competitors differ in their entry costs, but potential entrants have identical variable costs function. Yet, it looks at entry with a continuum of competitors. That paper, unlike this one, finds that fixed costs are always minimized with infinitesimal entrants that make sequential entry decisions. 
The paper proceeds as follows. In section 2, we describe the model. In section 3, we show industry size is invariant to entry ordering, and a necessary and sufficient condition for all entry orderings to be efficient is proved. In section 4 , we illustrate the results with a couple of examples. Finally, in section 5 the paper concludes. 


\section{[***Insert Figure 1 about Here***]}

\section{Model}

Let us begin by describing the game outlined in figure 1 above. The order by which firms of a given rank are allowed to enter is known by all firms in period -1 . Further, all previous entry decisions are common knowledge as they occur in period 0. In period 1, entrants compete without regard to their exogenous sunk costs. In period 1, all entrants receive an identical payoff before entry costs, which is only a function of the $N^{e}$ firms that enter. Let us define $\pi\left(N^{e}\right)$ as the payoff before entry costs for a unit-sized firm.

A firm that enters pays a sunk entry cost, $K_{i}$, which depends on the firm's rank $i$, where $i \in F=\{1,2,3, \ldots, n-1, n\}$. $^{2}$ The set of firms ranks $F \subset \mathbb{N}$. The number of elements of this set is $n(F)=n$, where $n \in \mathbb{N}$. The investment costs, $K_{i}$, are increasing in the firm's index number, $i$. That is, $K_{1}<K_{2}<K_{3}<\ldots<K_{n}$. Let us assume that per firm payoff, $\pi\left(N^{e}\right)$, declines in the number of entrants. That is, $\pi(1)>\pi(2)>\ldots>\pi(n$ $-1)>\pi(n) .^{3}$

We will assume that all firms that expect to make non-negative profits will enter. To ensure that it is subgame perfect Nash equilibrium (SPE) that at least one firm enters, let us assume that it is profitable for the lowest cost firm to always enter.

\footnotetext{
${ }^{2}$ Because entry costs are sunk, this paper precludes the possibility of the hit-and-run equilibria of contestable markets described by Baumol (1982).

${ }^{3}$ An example of a game with these properties would be a Cournot game where all entrants had identical variable cost functions. This example is pursued in section 4 and is derived in the appendix.
} 


$$
\pi(1)-K_{1} \geq 0
$$

Further, let us assume that if all firms enter, the highest cost firm in the set $F$ will find entry unprofitable.

$$
\pi(n)-K_{n}<0
$$

Suppose that the ordering of entry is a permutation of the set $F$. The entry set, $E$, consists of $n(E) \equiv N^{e}$ firms. If there is a potential entrant of rank $i$, it must be the case that $i \in F$. Further, if the firm of rank $i$ enters, $i \in E$. Since both $N^{e}$ and $n$ are integers, the set $E$ can consist of any ordering of the firms in set $F$ such that the $E \subseteq F$. There is an upper bound of

$$
M\left(n, N^{e}\right)=\frac{n !}{\left(n-N^{e}\right) !}
$$

possible permutations of entry orderings. Nevertheless, there will only be at most $C\left(n, N^{e}\right)$ different combinations of equilibrium entry sets for any random ordering of entry.

$$
C\left(n, N^{e}\right)=\frac{n !}{N^{e} !\left(n-N^{e}\right) !}
$$


Yet, there will only be one (unordered) entry set that is part of the unique subgame perfect Nash equilibrium (SPE) for any entry sequence.

\section{Analysis}

In this section we will prove two propositions regarding free entry and sunk cost efficiency when firms are of identical but discrete size. First, we demonstrate that the industry size does not depend on the entry ordering. Then, we will prove the necessary sufficient conditions to achieve sunk cost efficiency in the industry.

The implication of the inequalities in (1) and (2) is that some firms enter and some firms stay out of the industry. Let us define the free entry number of firms by the following set of conditions:

$$
\begin{aligned}
& \pi\left(N^{e}\right)-K_{N^{e}} \geq 0 \\
& \pi\left(N^{e}+1\right)-K_{N^{e}+1}<0
\end{aligned}
$$

\section{Proposition 1}

The ordering of potential firms will not affect the number of entrants.

From equation (5), we know that all firms with index numbers $0 \leq i \leq N^{e}$ will enter if no one else does. Further, these low index number firms, $i \leq N^{e}$, might even enter if the industry has greater than $N^{e}$ firms. To get more than $N^{e}$ entrants, it is necessary that the firm of rank $N^{e}+1$ or some higher ranked firm enters also. The 
free entry condition in (5) precludes this possibility because $\pi\left(N^{e}+1\right)<K_{N^{e}+1}$. If the firm with the index number $N^{e}+1$ will not enter an industry which will have $N^{e}+1$ entrants, then no other higher index number firm with an index number $N>N^{e}+1$ will enter under such circumstances. Q.E.D.

For any given equilibrium industry size, $N^{e}$, efficiency minimizes the entry costs incurred to reach that industry size. Therefore, efficiency under free entry depends on the identity of who enters — not just on the number of firms entering, $N^{e}$. (The latter problem was the only concern of Mankiw and Whinston (1986).) Total sunk cost of entry are $\sum_{\forall i \in E} K_{i}$. Since sunk costs are increasing in firms’ index numbers, sunk cost efficiency means that only the $N^{e}$ firms with the lowest index numbers, $i \leq N^{e}$, enter for a given industry size, $N^{e} .{ }^{4}$

\section{Proposition 2}

The necessary and sufficient conditions for all possible entry orderings to minimize entry costs for any equilibrium size, $N^{e}$, is $\forall j>N^{e}$ either
A) $\quad K_{j}>\pi\left(N^{e}\right)$, or
B) $\quad \forall i \leq N^{e}$, which could enter after $j, \pi\left(N^{e}+1\right) \geq K_{i}$

It is not hard to see that if we replaced any firm with rank $i \leq N^{e}$ with a firm of rank $j>N^{e}$ that the total entry costs would unambiguously rise because entry costs are strictly increasing in a firm’s rank.

\footnotetext{
${ }^{4}$ I would like to thank Antoine Faure-Grimaud and an anonymous referee for suggesting a ways to strengthen proposition 2.
} 
The proof for part A of proposition 2 is as follows. $K_{j}>\pi\left(N^{e}\right)$ implies that all firms of rank $j>N^{e}$ will stay out. That leaves only the potential entrants of rank $i \leq$ $N^{e}$. We know from the first inequality that all these firms will enter if that means that the size of the industry is equal to or less than $N^{e}$. This must be the case, and all firms of index numbers of $i$, where $i \leq N^{e}$, will enter. Q.E.D.

Now, we can consider part B in proposition 2. Suppose that $\pi\left(N^{e}\right)-K_{j} \geq 0$ for some firms of rank $j>N^{e}$. If all the $i$ ranked firms enter before a firm $j>N^{e}$, then the $j$-th firm will not enter because $\pi\left(N^{e}+1\right)-K_{j}<0$, according to the second inequality in the free entry conditions in equation (5). Yet, if the a firm of rank $i$ enters after a firm or rank $j$, then the $j$-th firm will only find entry profitable if it can deter firm $i$ from entering. Consider all the firms of rank $i \leq N^{e}$ that firm ranked $j^{*}=\left(N^{e}+1\right)$ could discourage from entering the industry. Firm $N^{e}+1$ will have the best chance of deterring the $N^{e}$-th firm from entry, because lower ranked firms have lower costs of entry. Yet, if firm $N^{e}+1$ enters before firm $N^{e}$, it cannot deter firm $N^{e}$ from entering by its entry alone if firm $N^{e}$ is profitable in an industry of $N^{e}+1$ firms. That is, if $\pi\left(N^{e}+1\right)-K_{N^{e}} \geq 0$, the entry of the $j^{*}$-th firm will not discourage any firm of rank $i$ from entering. Therefore, the ( $\left.N^{e}+1\right)$-th firm will stay out if $K_{N^{e}} \leq \pi\left(N^{e}+1\right)$, regardless of the entry permutation. Since lower ranked firms have lower entry costs, we can be sure that all firms of rank $i$ will enter if firm $j$ enters. That is, $K_{i} \leq \pi\left(N^{e}+\right.$ 1) for all $i \leq N^{e}$. Further, all higher $j$ firms, of rank higher than $j^{*}=N^{e}+1$, will also stay out, regardless of the entry ordering because the $\pi\left(N^{e}+1\right)<K_{N^{e}+1}<K_{N^{e}+2}<\ldots$ Q.E.D. 
If the necessary and sufficient conditions for proposition 2 are met, it is a unique subgame perfect Nash equilibrium (SPE) that all firms of rank $i \leq N^{e}$ will enter and all firms of rank $j>N^{e}$ will stay out regardless of entry ordering. 


\section{Two Examples}

Here we will explore two numerical examples. The Nash equilibrium outputs, producer surplus, consumer surplus and welfare for a given number of entrants are derived in the appendix. Firms are assumed to play a simultaneous Cournot game in period 1 .

For the examples in this section, the inverse demand intercept, $a$, inverse demand slope, $b$, and the marginal cost, $c$, parameters for both examples are

$$
\begin{aligned}
& a=10 \\
& b=1 \\
& c=0 .
\end{aligned}
$$

Under the parameters suggested in equation (6) above and the formula for the per firm payoff before entry costs derived in the appendix equation (12), we know that per firm producer surplus as a function of the number of entrants will be

$$
\begin{aligned}
& \pi(1)=25 \\
& \pi(2)=11 \frac{1}{9} \\
& \pi(3)=6.25 .
\end{aligned}
$$

Inserting the parameter values in (6) into the equation for equilibrium consumer surplus, which is derived in equation (15) in the appendix, consumer surplus is the following, depending on the number of entrants: 


$$
\begin{aligned}
& C S(1)=12.5 \\
& C S(2)=22 \frac{2}{9} \\
& C S(3)=28.125
\end{aligned}
$$

\subsection{Example 1}

$$
K_{1}=7, K_{2}=8 \text {, and } K_{3}=9 \text {. }
$$

Here free entry dictates that only two firms enter. $N^{e}=2$ as defined by equation (5). That is, $K_{2}=8<\pi(2)=11 . \overline{1}$, and $K_{3}=9>\pi(3)=6.25$. Yet, the most efficient entry set is not the only possible SPE when all entry orderings are equally likely. We know this because $K_{3}=9<\pi(2)=11 . \overline{1}$, and $\pi(3)=6.25<K_{2}=8$. Therefore, neither part of proposition 2 is satisfied. Indeed, there is no ordering where the $3^{\text {rd }}$ ranked firm is given the first or second opportunity to enter where the last firm, of either rank 1 or 2 , will credibly enter. That is, both $K_{1}=7>\pi(3)=6.25$ and $K_{2}=8>\pi(3)=6.25$. Therefore, if entry sequences are independent and identically distributed (i.i.d.) one-third of the time the first-best free entry set, $E_{1}=\{1$, 2 \}, will enter, generating social welfare of $29 . \overline{4}$; one-third of the time the second-best set of firms will enter, $E_{3}=\{1,3\}$, generating social welfare of $28 . \overline{4}$; and one-third of the time the worst set of firms will enter, $E_{2}=\{2,3\}$, generating social welfare of 
27. $\overline{4}$. Therefore, if entry orderings are i.i.d., then expected welfare is $28 . \overline{4}$ which is lower than $29 . \overline{4}$, the social welfare if the lowest ranked firms entered first. ${ }^{5}$

\subsection{Example 2}

$$
K_{1}=5, K_{2}=6 \text {, and } K_{3}=10 \text {. }
$$

Here, too free entry dictates that only two firms enter. That is, $K_{2}=6<\pi(2)$ $=11 . \overline{1}$, and $K_{3}=10>\pi(3)=6.25$. The $3^{\text {rd }}$ ranked firm with fixed costs $K_{3}=10$ would want to enter if there would only be two entrants, given that it entered. Yet, it would lose money if three firms entered. The $3^{\text {rd }}$ ranked firm cannot deter entry by the lower ranked firms. With these fixed costs for potential entrants, only the most efficient free entry equilibrium is possible. That is, the entry set $E_{1}=\{1,2\}$ is the unique SPE, regardless of entry ordering. We know this is the case because the sufficient condition from the part B of proposition 2 is met. Namely, $K_{3}=10<\pi(2)$ $=11 . \overline{1}$. Yet the $3^{\text {rd }}$ firm cannot deter entry of either the second or first firm because $\pi(3)=6.25>K_{2}=6>K_{1}=5$. Therefore, there is no entry permutation in which the lower ranked firms will not enter if the third ranked firm entered. Therefore, the $3^{\text {rd }}$ firm will never enter in equilibrium regardless of entry ordering. In this case, social welfare is $33 . \overline{4}$ under free entry or when entry is regulated by a social planner.

\footnotetext{
${ }^{5}$ Mankiw and Whinston (1986) argue that sometimes free entry leads to excessive entry. This is the case here. If entry was regulated by a social planner in this example, then only the $1^{\text {st }}$ ranked firm would be allowed to enter and social welfare would be 30.5. This is higher than the best free entry equilibrium in terms of welfare, which generated welfare of $29 . \overline{4}$.
} 


\subsection{Discussion of the Examples}

In each example, the set of potential entrants is and $F=\{1,2,3\}$. Therefore, we have three firms, $n(F)=n=3$, that are contemplating entry. In both examples, we find that two firms, $N^{e}=2$, enter the industry in equilibrium. Nevertheless, welfare would always be weakly higher under free entry if the entry order was such that the lowest fixed cost firms moved first.

In both examples, two firms find entry optimal. Yet, in the first example, there are $C\left(n, N^{e}\right)=C(3,2)=3$ potential subgame perfect Nash equilibrium (SPE) entry combinations $-E_{1} \equiv\{1,2\}, E_{2} \equiv\{2,3\}$, and $E_{3} \equiv\{1,3\}$. In contrast, in the second example, the $3^{\text {rd }}$ ranked firm cannot deter the entry of the $2^{\text {nd }}$ ranked firm. Therefore, as in proposition 2, the most efficient free entry equilibrium combination- $E_{1} \equiv\{1,2\}$ —is the SPE regardless of entry ordering.

Both these examples illustrate the concept that ordering does not affect the number of entrants when competitors have identical payoffs after entry, $\pi\left(N^{e}\right)$, proposition 1 . Nevertheless, ordering does potentially affect welfare when firms are discrete as higher fixed cost competitors may enter and preclude lower fixed cost firms from entering. The necessary and sufficient condition for entry ordering irrelevance in proposition 2 is supported by these examples as well. 


\section{Conclusion}

This paper has considered an entry game in which firms have identical payoffs upon entry but differ in their sunk entry costs. In this game, both the exogenously given cost functions and entry orderings are common knowledge. Under these conditions, entry ordering cannot affect the size of the industry or the number of competitors. Yet, entry orderings can sometimes affect the sunk costs in an industry. With discrete competitors, higher fixed cost entrants can potentially block lower fixed cost potential competitors from entering. A necessary and sufficient condition for all entry orderings to minimize fixed costs is derived. 


\section{References}

Baumol, William J., 1982, “Contestable Markets: An Uprising in the Theory of Industrial Structure,” American Economic Review, 72, 1-15.

Dixit, Avinish, 1980, “The Role of Investment in Entry Deterrence,” Economic Journal, 90, 95-106.

Ghemawat, Pankaj, and Nalebuff, Barry, 1985, “Exit,” The Rand Journal of Economics, 16, 184-194.

Mankiw, Gregory, and Micheal D. Whinston, 1986, “Free Entry and Social Inefficiency,” Rand Journal of Economics, 17, 48-58.

Roberts, Kevin, 2007, “The Participant's Curse and the Perception of Unequal Treatment,” Economics Letters, 97, 162-169.

Spence, Michael, 1977, “Entry, Capacity, Investment and Oligopolistic Pricing,” Bell Journal of Economics, 12, 49-70.

Wilson, Linus, 2009, "Fixed Cost Efficiency with Infinitesimal Competitors,” forthcoming Applied Economics Letters. 


\section{Appendix: Deriving the Linear Cournot Model}

Here we derive the linear Cournot model which is used to analyze the examples in section 4. $q\left(N^{e}\right)$ is per firm output, which is a function of the number of entrants, $N^{e}$. Total industry output is $N^{e} q\left(N^{e}\right) \equiv Q$. Inverse demand is defined as price as function of industry output, $P(Q)$. Suppose that all firms are identical Cournot competitors who face a linear inverse demand curve $P(Q)=a-b Q$. Further, all competitors have identical cost functions $c(q)=c q\left(N^{e}\right)$, where $c \geq 0$ is the marginal cost parameter. Firms are assumed to play a simultaneous move Cournot game in period 1.

The Nash equilibrium per firm output for an industry with $N^{e}$ identical competitors is

$$
q\left(N^{e}\right)=\frac{a-c}{b\left(N^{e}+1\right)}
$$

Total industry output in equilibrium is

$$
Q\left(N^{e}\right) \equiv N^{e} q\left(N^{e}\right)=\frac{N^{e}}{N^{e}+1} \frac{a-c}{b} .
$$

The equilibrium price is 


$$
P\left(N^{e}\right)=\frac{a+N^{e} c}{N^{e}+1}
$$

Per firm producer surplus for all entrants is

$$
\pi\left(N^{e}\right)=\left(\frac{a-c}{N^{e}+1}\right)^{2} \frac{1}{b} .
$$

If the $i$-th firm enters, its profits after sunk costs are

$$
\pi\left(N^{e}\right)-K_{i}=\left(\frac{a-c}{N^{e}+1}\right)^{2} \frac{1}{b}-K_{i} .
$$

Total profits for the industry are industry producer surplus, or payoff before sunk costs, $\Pi\left(N^{e}\right)$, less total investment costs, $\sum_{\forall i \in E} K_{i}$. That is, industry profits are

$$
\Pi\left(N^{e}\right)-\sum_{\forall i \in E} K_{i}=\left(\frac{a-c}{N^{e}+1}\right)^{2} \frac{N^{e}}{b}-\sum_{\forall i \in E} K_{i} .
$$

Total consumer surplus, $C S\left(N^{e}\right)$, generated by this industry is

$$
C S\left(N^{e}\right)=\frac{b Q^{2}}{2}=\left(\frac{N^{e}(a-c)}{N^{e}+1}\right)^{2} \frac{1}{2 b} .
$$


Therefore total welfare, $W\left(N^{e} ; E\right)$, which is a function of both the size of the industry, $N^{e}$, and the entry set, $E$, is

$$
\begin{aligned}
& W\left(N^{e} ; E\right) \equiv \Pi\left(N^{e}\right)+C S\left(N^{e}\right)-\sum_{\forall i \in E} K_{i} \\
& =\left(\frac{a-c}{N^{e}+1}\right)^{2} \frac{N^{e}}{b}\left(1+\frac{N^{e}}{2}\right)-\sum_{\forall i \in E} K_{i} .
\end{aligned}
$$


Ordering of potential entrants is determined and becomes common knowledge.

Figure 1: Sequence of Events
- Firms sequentially choose to enter.

-Entrants pay the sunk costs of entry.
$N^{e}$ entrants each earn a payoff of $\pi\left(N^{e}\right)$. 